\title{
L'anfora «Dressel 26» Del Castro Pretorio
}

\author{
Alessia Contino*, Claudio Capelit ${ }^{* *}$, Marina Milella ${ }^{* * *}$, Francesco PaCetti ${ }^{* * *}$, \\ Lucrezia UNGARO ${ }^{* * *}$, Michel BONIFAY ${ }^{* * * *}$
}

Parole chiave: Anfore; Castro Pretorio; Roma; Dressel; Tipologia; Epigrafia; Petrografia; Africa.

Riassunto: Grazie alla collaborazione tra la Sovraintendenza Capitolina ai Beni Culturali e il Centre Camille Jullian è stato possibile effettuare una caratterizzazione di dettaglio dell'anfora Dressel 26 proveniente dal Castro Pretorio a Roma. Tale contenitore, individuato da H. Dressel nel 1879, non era stato finora oggetto di studi sistematici. Solo recentemente sono state proposte alcune ipotesi circa l'interpretazione del corredo epigrafico e le possibili indicazionisulcontenutoel'originedell'anfora. Irisultatidella presente ricerca, integrando $i$ dati archeologici e petrografici, suggeriscono una provenienza dall'Africa settentrionale e non dalla Sicilia orientale o dall'Italia meridionale, come proposto in precedenza.

Keywords: Amphorae; Castro Pretorio; Rome; Dressel; Typology; Epigraphy; Petrography; Africa.

Abstract: The collaboration between Sovraintendenza Capitolina ai Beni Culturali and Centre Camille Jullian allowed the Dressel 26 amphora from Castro Pretorio in Rome to be characterised in detail. Systematic studies on this container, identified by H. Dressel in 1879, have not been carried out yet. Only recently a few hypotheses on epigraphy, content and provenance of amphorae Dressel 26 have been proposed. The results of our research, based on the combination of archaeological and petrographic data, point to a possible origin from North Africa and neither from Eastern Sicily or Southern Italy, as previously suggested.

Mots-clefs : amphores ; Castro Pretorio ; Rome ; Dressel ; typologie ; épigraphie; pétrographie ; Afrique.

Resumé : Dans le cadre d'une collaboration entre la Sovraintendenza Capitolina ai Beni Culturali et le Centre Camille Jullian, une caractérisation détaillée de l'amphore Dressel 26 provenant du Castro Pretorio à Rome a été menée à bien. Ce type de conteneur, identifié par H. Dressel en 1879, n'avait pas fait l'objet jusqu'ici d'études systématiques et c'est seulement récemment que des hypothèses sur l'épigraphie, le contenu et la provenance de cette amphore ont pu être proposées. Les résultats de la présente recherche suggèrent une origine en Afrique du Nord et non pas en Sicile ou en Italie du Sud comme cela avait été précédemment proposé.

\section{INTRODUZIONE}

\section{La collezione Dressel e i Mercati di Traiano}

Il suggestivo ambiente di una cisterna rinascimentale, attualmente accessibile dalla Grande Aula dei Mercati di Traiano, e la ricca collezione di anfore romane che vi erano depositate sono stati oggetto di un complesso intervento di restauro delle strutture e dei materiali (2013-2014), che ha consentito di aprire al pubblico una nuova sala del Museo dei Fori Imperiali, ampliando l'esposizione con materiale di grande interesse ${ }^{1}$.

\footnotetext{
* Soprintendenza Speciale per il Colosseo e l'Area archeologica centrale di Roma, collaboratrice associata al Centre Camille Jullian (Aix Marseille Univ, CNRS, MCC, CCJ, Aix-en-Provence, France), alessia.contino@beniculturali.it (A.C.).

** Dipartimento di Scienze della Terra, dell'Ambiente e della Vita (DISTAV), Università degli Studi di Genova, Gênes, Italie. Collaboratore associato al Centre Camille Jullian (Aix Marseille Univ, CNRS, MCC, CCJ, Aix-en-Provence, France), capelli@dipteris.unige.it (C.C.).
}

Il nucleo principale della collezione consiste nelle anfore rinvenute nel 1878 nel deposito di Castro Pretorio e studiate da Heinrich Dressel (di cui 160 con iscrizioni) ${ }^{2}$, al quale si sono aggiunti esemplari di diversa provenienza, depositati nei Mercati di Traiano in più fasi a partire dagli anni Trenta del secolo scorso, per un totale di 480 esemplari, in maggioranza interi. L'integrità dei reperti, la varietà delle forme e le necessità della conservazione hanno giustificato il recente allestimento nella cisterna rinascimentale all'interno del monumento.

*** Sovraintendenza Capitolina ai Beni Culturali, marina.milella@ comune.roma.it (M.M.), francesco.pacetti@comune.roma.it (F.P.), lucrezia.ungaro@comune.roma.it (L.U.).

**** Directeur de Recherche au CNRS, Centre Camille Jullian (Aix Marseille Univ, CNRS, MCC, CCJ, Aix-en-Provence, France), mbonifay@mmsh.univ-aix.fr (M.B.).

1. UNGARO et alii 2014.

2. DRESSEl 1879. 
Oggi il corredo epigrafico delle anfore di Castro Pretorio ha perso la maggioranza dei tituli picti, molti dei quali erano già in precario stato di conservazione al momento della scoperta.

Tra il 2013 ed il 2014 è stato eseguito il restauro di 151 anfore, 127 delle quali sono state esposte nel nuovo allestimento. Durante l'intervento, la presenza di tituli picti di pigmento scuro (nero e rosso), non visibili in precedenza a causa dei depositi e delle incrostazioni, ha richiesto un approfondimento con tecniche di indagine scientifica ${ }^{3}$. È stato possibile identificare sul totale degli esemplari 60 bolli, 15 tituli picti, 7 graffiti e 5 contrassegni.

L'opportunità di lavorare in maniera contestuale su un gran numero di anfore ha permesso di acquisire nuove conoscenze dal punto di vista sia conservativo che scientifico, grazie anche alla collaborazione tra diverse professionalità (archeologi, restauratori, architetti, fisici) e ha ampliato la conoscenza dei materiali fornendo ulteriori dati utili alla comprensione e ad una futura gestione dei manufatti.

Il criterio seguito nell'allestimento risponde al principio della storicizzazione della collezione, mettendone in risalto il valore documentario e sottolineando la portata storica degli studi di Dressel.

Di qui la scelta di riprodurre materialmente, purtroppo solo in parte a causa della dispersione di alcuni esemplari avvenuta nel corso del tempo, la tavola tipologica del CIL sulla parete di fondo della cisterna (fig.1), destinando i due lati lunghi dell'ambiente a una panoramica del repertorio dei principali tipi anforici di produzione italica (fig. 2) e iberica (fig. 3), per la maggior parte provenienti dal deposito di Castro Pretorio.

Nei casi in cui è stato possibile istituire un collegamento diretto tra l'apparato epigrafico edito nel CIL e un'anfora specifica, questa è stata inserita nella ricostruzione della tavola di Dressel rispettando la sequenza adottata dallo studioso nella sua classificazione morfologica.

È questo il caso della Dressel 26 inv. MT 351 (CIL XV, 3479a) oggetto dello studio che segue.

(M.M., F.P., L.U.)

\section{Il protocollo d'intesa tra il Centre Camille Jullian e la Sovrintendenza Capitolina al Beni Culturali}

La ricerca dottorale sulle produzioni africane di età tardo repubblicana e primo imperiale a partire dai dati del sito romano del Nuovo Mercato Testaccio, di recente conclusa ${ }^{4}$, è stata l'occasione per istituire una proficua collaborazione tra il Centre Camille Jullian (Aix-Marseille Univ, CNRS, MCC, CCJ, F-13000, Aix-en-Provence, France) e la Sovrintendenza Capitolina ai Beni Culturali, con lo scopo di effettuare

3. I restauri delle anfore sono stati eseguiti da Massimiliano Massera e Laura Rivaroli, con la supervisione di Cecilia Bernardini. Sono state eseguite, in collaborazione con il Dott. Fabio Aramini dell'ISCR, indagini scientifiche volte alla ricerca sperimentale di individuazione di tituli picti più visibili mediante riflettografia. Lo studio del corredo epigrafico è di Paolo Vigliarolo.

4. Contino 2015. ricerche comuni su alcune anfore di produzione africana, tra cui il contenitore oggetto del contributo. Il protocollo d'intesa prevede la possibilità di effettuare campionamenti petrografici e nuova documentazione grafica e fotografica di alcuni esemplari, particolarmente rappresentativi per il loro valore documentale e storico. È questo il caso dell'anfora eponima del tipo Dressel 26 o di alcuni contenitori di più recente individuazione di cui il deposito dei Mercati Traianei vanta esemplari integri, come il tipo tardo Keay 3/5, di cui si suppone la produzione nella zona di Nabeul, attualmente in corso di studio ${ }^{5}$.

La possibilità di far convergere l'impegno e l'interesse comune su depositi di così grande valore storico costituisce un momento fondamentale nella crescita degli studi attuali, grazie anche all'applicazione di nuove metodologie di ricerca agli esemplari conservati in tali depositi. Lo studio qui presentato costituisce il felice risultato di questa collaborazione che ci si augura proficua anche in futuro.

(M.M., F.P., L.U., M.B.)

\section{IL TIPO DRESSEL 26}

\section{StORIA DeGli STUDI}

L'anfora eponima del tipo Dressel 26 venne identificata da Heinrich Dressel nel deposito del Castro Pretorio negli anni '70 dell' 800 . Al tipo, collocato nella tavola al numero 26 (fig. 4), furono assegnati nel CIL due esemplari con bollo sul collo: uno integro, ancora oggi visibile presso i depositi dei Mercati di Traiano, e uno frammentario, al momento non reperibile ${ }^{6}$.

L'anfora compare a Pompei sotto il tipo Schoene $\mathrm{XI}^{7}$. Tuttavia tale forma, come dimostrato da C. Panella nel contributo sulle anfore tripolitane di Pompei del 1977, riuniva almeno quattro diversi contenitori, poi rinvenuti dalla studiosa nei depositi pompeiani ${ }^{8}$. Un primo tipo è rappresentato dall'anfora nota nel catalogo romano del Dressel come Dressel 26, un secondo tipo è il contenitore denominato Ostia LIX dalla stessa Panella nei contesti delle Terme del Nuotatore", vi è poi un tipo di "grande modulo" con una morfologia dell'orlo simile al precedente ${ }^{10}$, infine vi è la Tripolitana I che rappresenta il contenitore di riferimento e numericamente più attestato per il tipo XI di Schoene (fig. 5). Lo Schoene prima e il Mau successivamente avevano infatti proceduto ad una classificazione dei contenitori anforici su base epigrafica, con una scarsissima sensibilità

\footnotetext{
5. Pubblicazione in preparazione.

6. Dressel 1879, p. 190-191; CIL XV, 3479.

7. CIL IV, che raccoglie l'instrumentum domesticum di Pompei e organizza i contenitori da trasporto in 45 forme alle quali riconduce la quasi totalità dei ritrovamenti.

8. Panella 1977, p. 141-143.

9. Carandini, Panella (éd.) 1973, fig. 386.

10. Panella 1977, fig. 33a e b.
} 


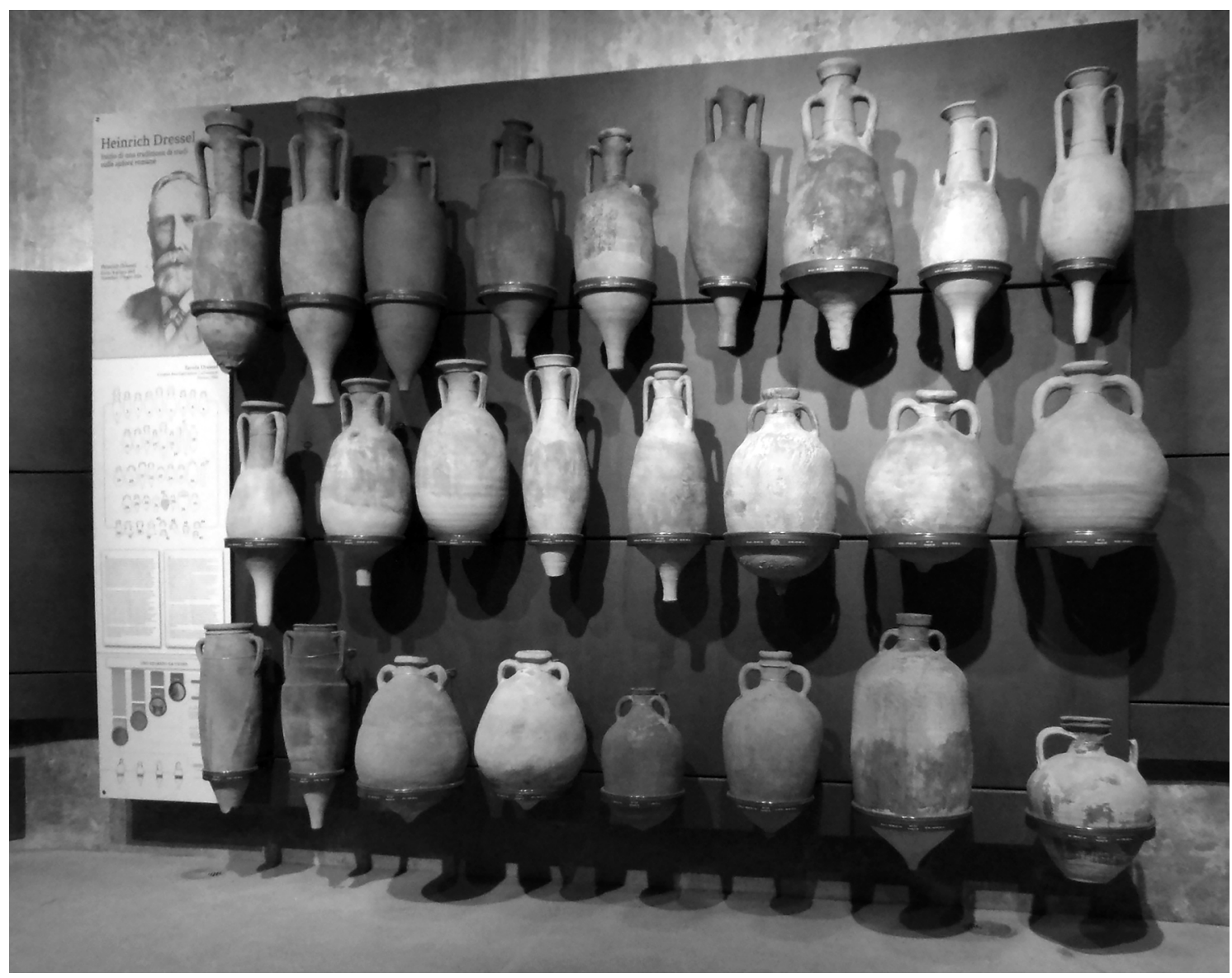

Fig. 1 : Le anfore della tavola Dressel esposte nel Museo dei Fori Imperiali nei Mercati di Traiano (Roma).

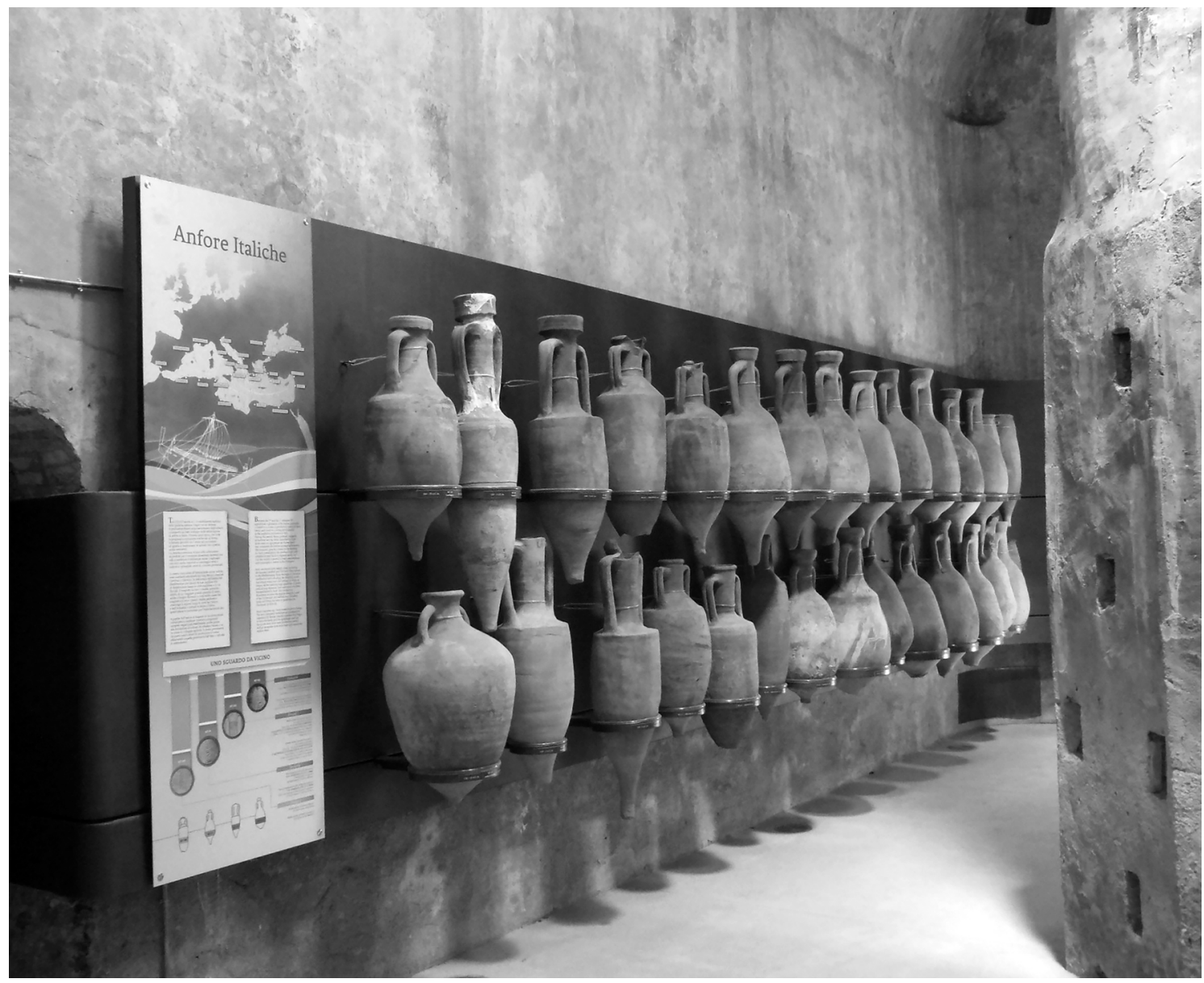

Fig. 2 : Le anfore di produzione italica esposte nel Museo dei Fori Imperiali nei Mercati di Traiano (Roma). 


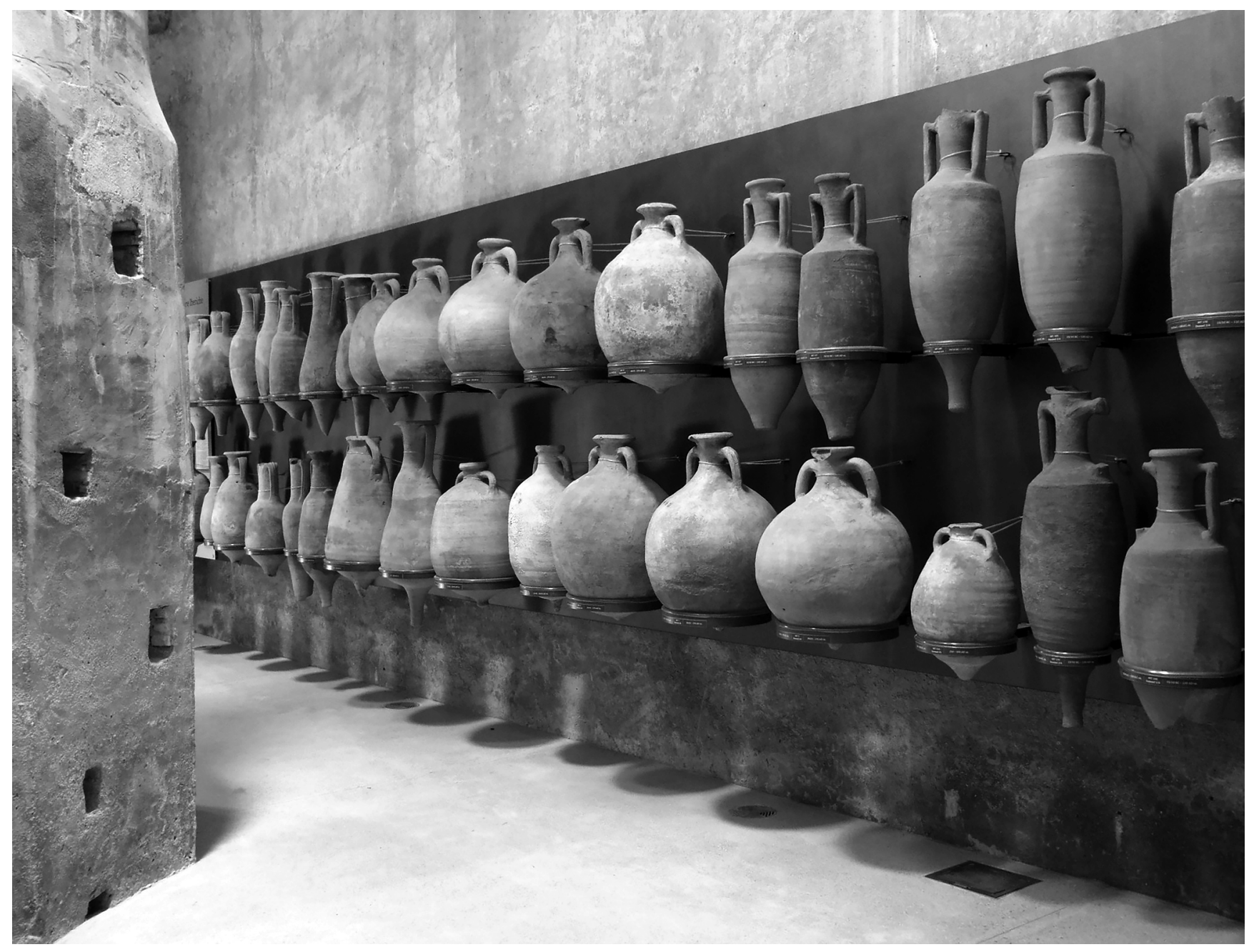

Fig. 3 : Le anfore di produzione iberica esposte nel Museo dei Fori Imperiali nei Mercati di Traiano (Roma).

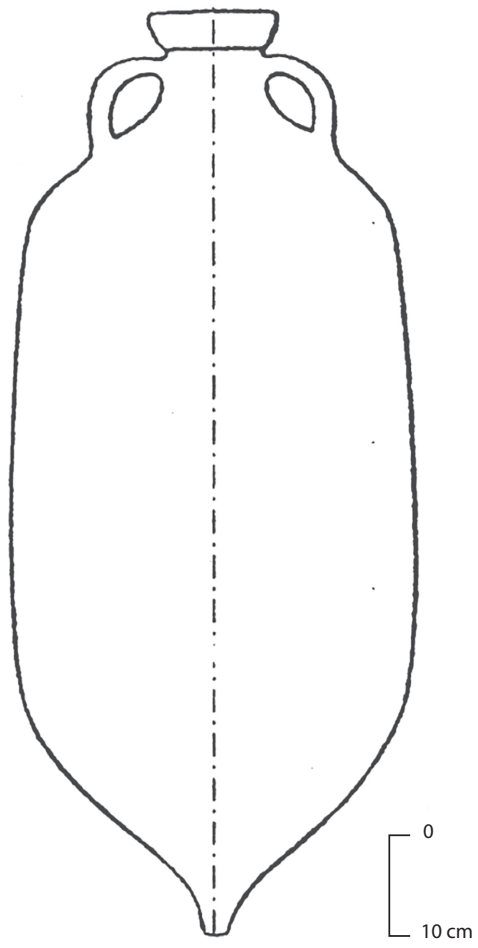

Fig. 4 : Anfora Dressel 26 eponima. Roma, Castro Pretorio (Dressel 1879) 


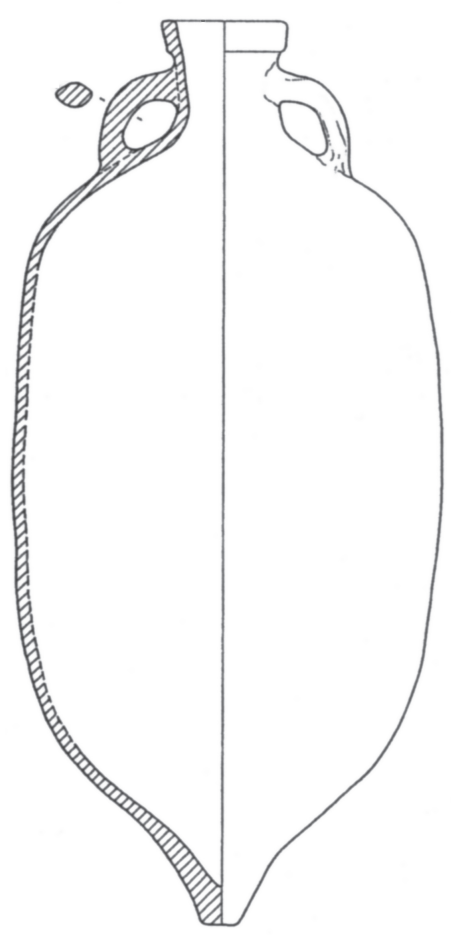

34 b

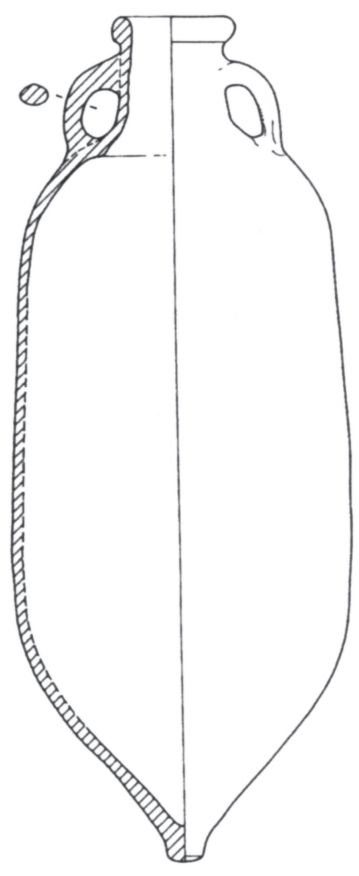

32 b

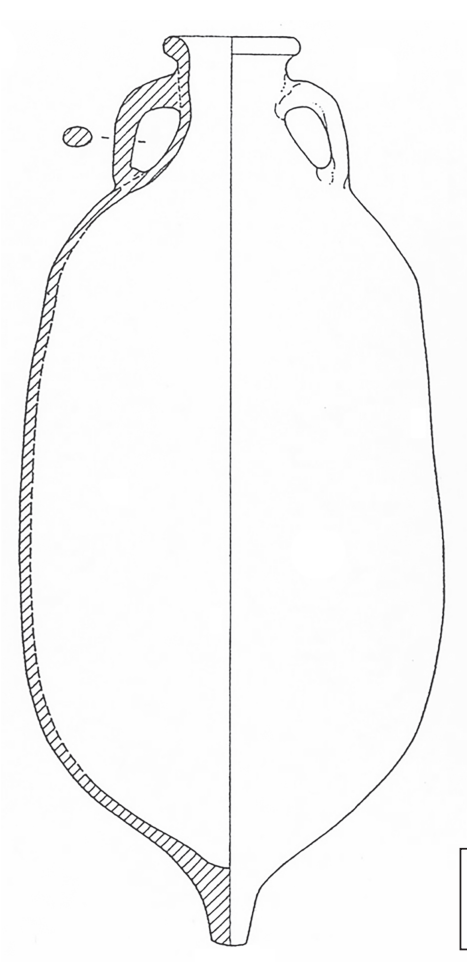

$33 b$

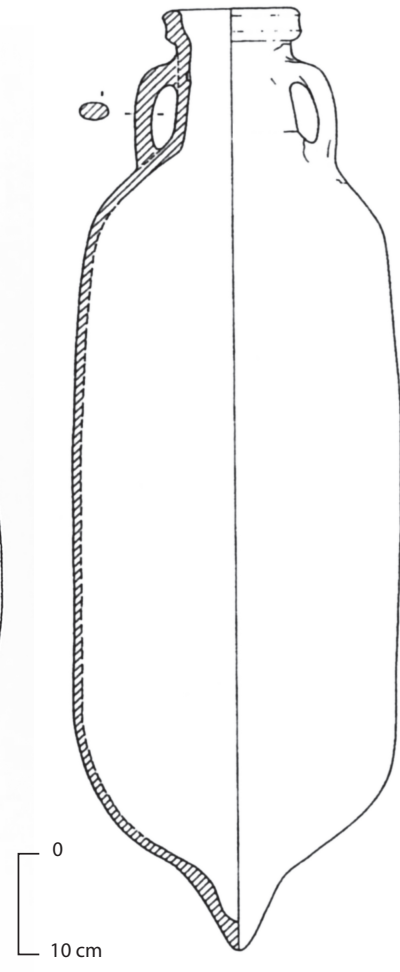

$8 b$

Fig. 5 : Anfora tipo Schoene XI. Pompei. 1) Dressel 26 (PANella 1977, tav. LXVII, 34b); 2) Ostia LIX (PANeLla 1977, tav. LXVI, 32b); 3) Tipo Panella 1977, tav. LXVII, 33b; 4) Tripolitana I (PAnella 1977, tav. LXI, 8b).

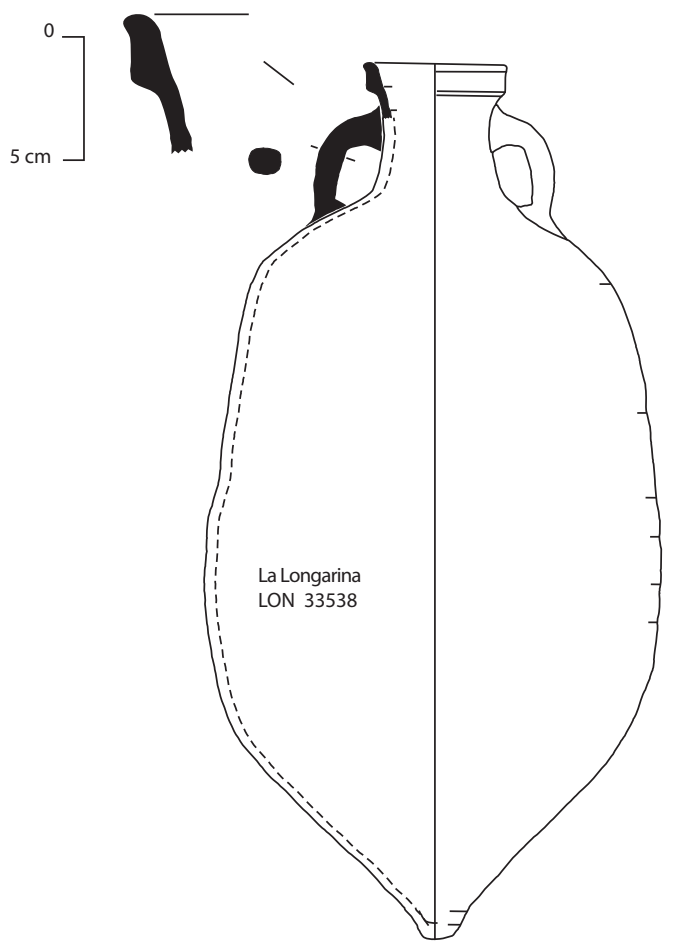

1

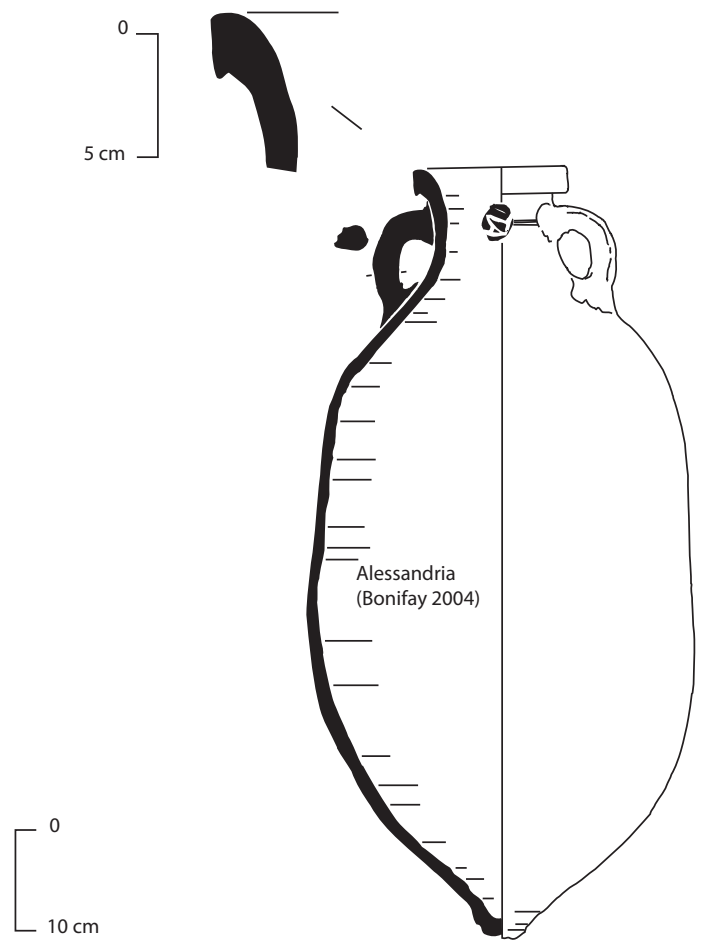

2

Fig. 6 : Esempi di Anfore Africane Antiche in precedenza identificate come Dressel 26: 1) La Longarina, Inv. 33538 (disegno A. Contino); 2) Alessandria (BONIFAY 2004). 

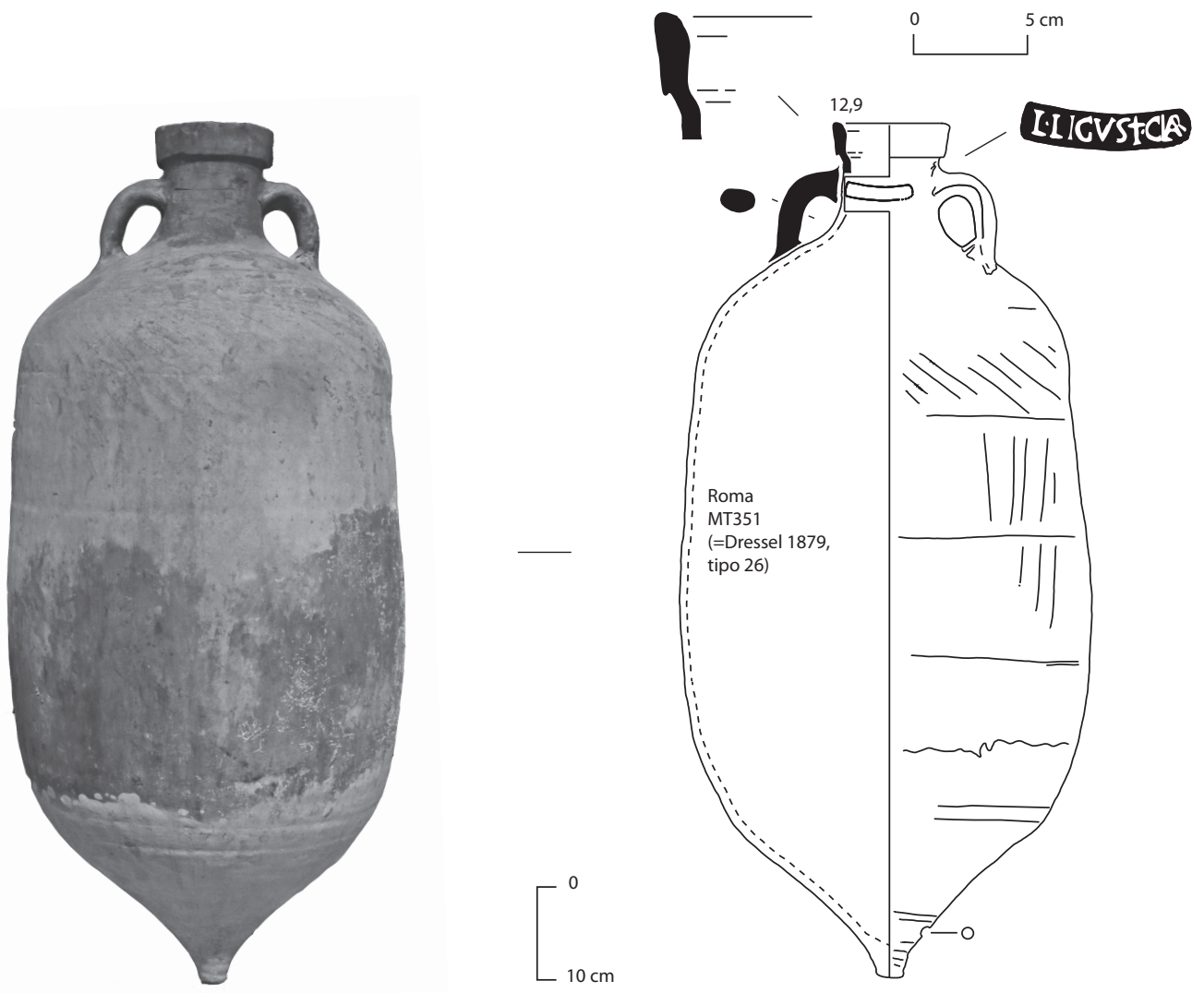

Fig. 7 : Anfora Dressel 26 eponima. Roma, Castro Pretorio. Mercati di Traiano, Inv. MT351 (foto M. Bonifay; disegno M. Bonifay, A. Contino).

per le questioni tipologiche, al contrario di quanto effettuato da H. Dressel al Castro Pretorio.

L'anfora sarebbe stata poi erroneamente segnalata da A. Hesnard nei contesti augustei della Longarina ${ }^{11}$. In realtà il deposito della Longarina rappresenta uno dei contesti ove è maggiormente attestato con esemplari integri un tipo di anfora africana di età tardorepubblicana e primo imperiale nota come Africana Antica ${ }^{12}$. Al momento dello studio della Hesnard tuttavia il tipo non era stato ancora individuato e l'unica forma di riferimento per questi contenitori era l'anfora Dressel 26.

Il contenitore è stato successivamente descritto da Empereur e dalla stessa Hesnard nel 1987 nel noto articolo sulle anfore di età ellenistica ${ }^{13}$, in cui si rilevava la difficoltà di identificare e differenziare proprio i due tipi, Africana Antica (ex "Tripolitana Antica") e Dressel 26.

Sulla scorta di questi lavori il libro di Sciallano e Sibella del $1991^{14}$, illustrando le anfore "Tripolitane antiche", associa nella stessa tavola la Dressel 26 del Castro Pretorio, una Tripolitana I precoce e una Africana Antica, entrambe provenienti dalla Longarina.

Questa articolata vicenda legata alla definizione del tipo ha provocato difficoltà ed errori di identificazione evidenti specialmente nei contesti romani, forse maggiormente influenzati dai ritrovamenti ostiensi della Longarina (fig. 6.1).

11. HeSNARD 1980, p. 150, pl. 7, 1, 2.

12. Contino 2013; Capelli, Contino 2013; Contino, Capelli c.s.

13. EMPEREur, Hesnard 1987, p. 35-36.

14. Sciallano, Sibella 1991.
In particolare si rammentano un frammento di anfora da Vigna Barberini in contesti di età flavia ${ }^{15}$; un'anfora intera da scavi di emergenza in Via Nelli ${ }^{16}$; un frammento di anfora proveniente dallo scavo di piazza Vittorio ${ }^{17}$; un'anfora proveniente dai contesti augustei dello scavo delle pendici del Palatino ${ }^{18}$.

Anche fuori da Roma esistono alcuni errori di identificazione: a titolo di esempio un'anfora da Alessandria d'Egitto ${ }^{19}$ (fig. 6.2) e una da Arles ${ }^{20}$.

Un articolo di F. Zevi del 1966, tra i primi, richiamava il tipo Dressel 26 attribuendo all'anfora un'origine italica, ed in particolare una provenienza da Venafro, soprattutto sulla base della sua presenza documentata esclusivamente a Roma e Pompei ${ }^{21}$. Successivamente all'articolo di F. Zevi, Theodor Peña ha proposto in uno studio del 2007 l'ipotesi di una provenienza siciliana, ed in particolare tauromenitana ${ }^{22}$.

(A.C.)

15. Rizzo 2003, pl. XXXVII, p. 197.

16. Lissi CARONNA 1968, p. 10-15, fig. 5a.

17. Ferrandes 2014, p. 363.

18. Tesi della Dott.ssa Diana Greco, Prof. Clementina Panella.

19. Bonifay 2004, p. 98, fig. 52, p. 101.

20. Long 1994, pl. 4, p. 66. È stato possibile visionare i disegni grazie alla cortese disponibilità del Dott. Guillaume Duperron.

21. Zevi 1966, p. 224-225.

22. PeÑa 2007. Sulla base di considerazioni epigrafiche che avremo modo di verificare a breve. 


\section{La Dressel 26 del Castro Pretorio: TIPOLOGIA, EPIGRAFIA, PETROGRAFIA, CONTENUTO}

\section{TIPOLOGIA}

Grazie alla collaborazione formalizzata con la Sovrintendenza Capitolina ai Beni Culturali è stato possibile accedere ai depositi dei Mercati di Traiano, ove è stata rinvenuta l'anfora integra identificata da $\mathrm{H}$. Dressel con il tipo 26 (MT 351). L'anfora, allora in corso di restauro per poter essere esposta nel nuovo allestimento da poco inaugurato nella sede dei Mercati di Traiano, è stata da noi disegnata direttamente all'interno dei depositi. In tale occasione è stato anche prelevato un campione di impasto dal contenitore.

L'anfora MT 351 ha un orlo a fascia molto regolare nella faccia esterna, probabilmente lisciato con l'ausilio di una stecca, con una leggera gola all'interno, un collo corto cilindrico che si restringe leggermente all'attacco con il bordo, anse a fascia con nervature e digitature all'attacco inferiore, sulla superficie esterna. Il corpo è cilindrico sagomato. Ha un fondo di forma conica, vuoto, a base piatta. Le dimensioni dell'anfora sono: H. $94 \mathrm{~cm}$ (H. interna 90,3 $\mathrm{cm}$ ); diam. max. $45 \mathrm{~cm}$; diam. orlo $12,9 \mathrm{~cm}$.

Osservando il contenitore è possibile individuare le tracce dell'assemblaggio dei diversi cilindri utilizzati per il corpo: oltre alla sezione del fondo e a quella della parte superiore a partire dalla spalla, si individuano almeno due cilindri per il ventre, di circa $13,5 \mathrm{~cm}$ di altezza ${ }^{23}$. L'anfora presenta inoltre steccature oblique tra le spalle e verticali lungo il corpo. Subito sopra il fondo è presente un foro probabilmente praticato per lo svuotamento. Uno schiarimento superficiale è presente dalla spalla al fondo del contenitore, mentre è assente sul collo; all'altezza dell'attacco inferiore delle anse sono presenti due circoli non schiariti. Tali tracce lasciano supporre che la cottura sia avvenuta in modo tale che il collo non fosse direttamente esposto al calore. I due circoli rossi in corrispondenza delle anse potrebbero suggerire la presenza di un supporto o di un oggetto che copriva il collo e si appoggiava alle spalle dell'anfora. Non siamo tuttavia in grado di proporre una ricostruzione sicura. Il fondo presenta uno sbiancamento molto evidente e spesso con tracce di gocciolatura (fig. 7).

L'anfora del Castro Pretorio risulta avere un modulo leggermente inferiore in altezza rispetto alla buona parte delle anfore dello stesso tipo documentate a Pompei ed in particolare un collo più corto e un fondo cavo di forma conica, molto simile a quelli normalmente attestati per le anfore Africane Antiche. Potrebbe forse trattarsi di una variante precoce del tipo ${ }^{24}$, poiché il deposito del Castro Pretorio è più antico di circa un ventennio rispetto alla distruzione di Pompei, con elementi morfologici di

23. Sulle modalità tecniche di realizzazione dei contenitori si veda tra gli altri Bonifay 2004, p. 44.

24. Per quanto attiene alla datazione del deposito del Castro Pretorio non oltre il 50/60 d.C. si vedano: Zevi 1966, p. 211-212; PAnella 1977, p. 143. transizione fra i due contenitori nelle loro varianti classiche. L'anfora si evolverebbe poi in forme a più spiccato profilo cilindrico con fondi di dimensioni maggiori, colli più lunghi e verticali e conseguentemente anse più lunghe e a fascia. Le misure medie dell'anfora nella sua variante di maggiori dimensioni sono infatti: H. 98-1,01 cm; diam. max. 41-44 cm; diam. orlo 12-13,5 cm; capacità media: 86 litri (fig. 8).

Le specificità morfologiche dell'anfora di Castro Pretorio, associate a quelle dell'impasto (v. infra), potrebbero altresì essere collegate ad una specifica variante produttiva e non necessariamente legata allo sviluppo cronologico del contenitore.

\section{Cronologia}

L'anfora non è molto diffusa nel Mediterraneo, ma è stato possibile verificare la sua presenza esclusivamente in contesti datati al I secolo d.C., in particolare a Roma presso il Nuovo Mercato Testaccio e nello scavo di Via Sacchi, a Ostia presso la taberna dell'Invidioso e la domus dei Pesci, a Pompei e nel deposito del relitto Arles-Rhône $3^{25}$. Tali contesti sono cronologicamente compatibili con il deposito del Castro Pretorio da cui proviene l'esemplare oggetto dello studio. La cronologia proposta quindi si colloca tra gli inizi e la fine del I secolo d.C.

\section{BoluI}

L'apparato epigrafico noto per l'anfora Dressel 26 proviene esclusivamente dalle attestazioni del Castro Pretorio dove erano documentati due esemplari bollati, di cui resta solamente il contenitore integro. L'esemplare integro riporta un bollo rettangolare sul collo «L. LIGVSTI CLAR» (fig. 9), mentre il secondo esemplare, oggi non rintracciabile, recava due bolli rettangolari sui due lati del collo «L. LIGVSTI» $\mathrm{e}$ «CLARI $»^{26}$.

Il gentilizio Ligustius nelle fonti è attestato sempre con il prenome Caius, tranne che nel caso delle anfore del Castro Pretorio ed è presente in Italia, oltre che a Roma nel I sec. d.C., a Palestrina, Capua e Venafro, ma in questi casi si tratta di epigrafi di difficile datazione. Si ritrova anche in Dalmazia a Salona in epigrafi funerarie. In questo caso la presenza della formula Dis Manibus nella forma abbreviata DM in una delle due iscrizioni potrebbe forse indicare una data successiva all'età flavia, probabilmente nel II d.C., ma non esclude una

25. Per il I d.C. si segnalano gli esemplari noti dalla bibliografia provenienti da Roma. NMT: Contino 2013; Gianicolo: Ferrandes 2008, p. 253. Esemplari provenienti da Ostia: taberna dell'invidioso: CARTA, PoHL, Zevi 1978, p. 93, fig. 97, n 119; domus dei pesci: Geremia NuCCI 2004-2005, p. 96, fig. 401; Rizzo 2014, p. 273, tab. 37. Esemplari provenienti da Pompei: Panella 1977, p. 142, tav. LXVII, fig. 34 a-b, 35 SCOTTI 1984, tav. 165. CE 2017, Casa di Arianna, inedito; si ringrazia per l'informazione il Dott. Albert Ribera; esemplare proveniente dal deposito del relitto Arles-Rhône 3, che abbiamo potuto esaminare grazie alla cortese disponibilità del Dott. David Djaoui.

26. DresSEl 1879, p. 190-191; CIL XV, 3479. 


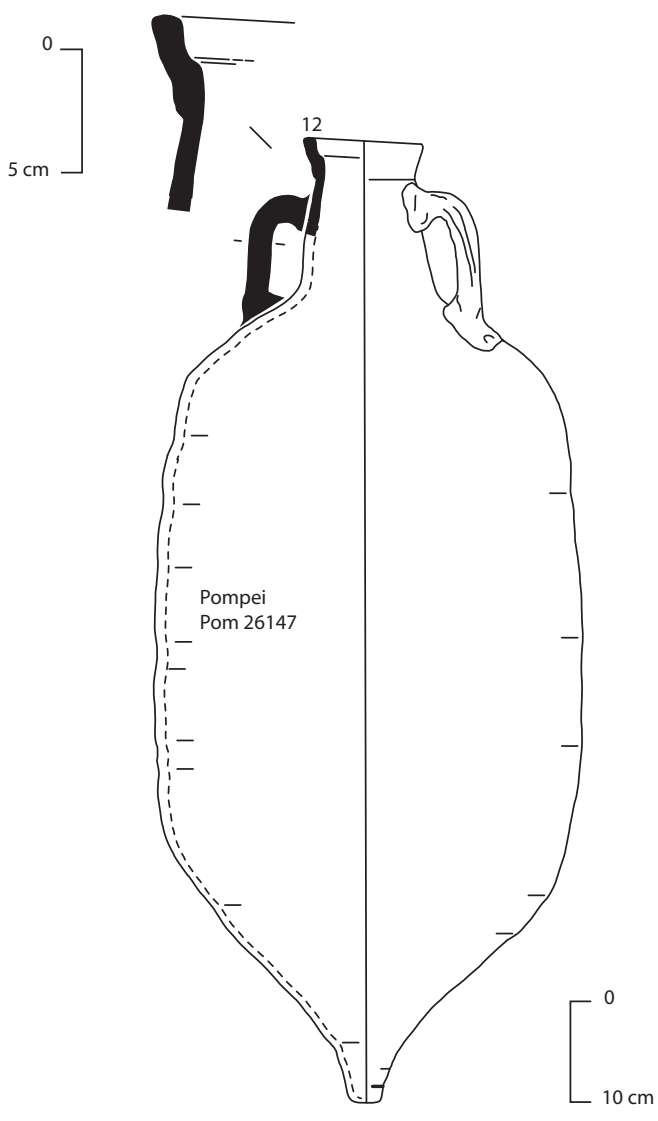

Fig. 8 : Anfora Dressel 26. Pompei. Granai del Foro, Inv. 26147 (disegno A. Contino).
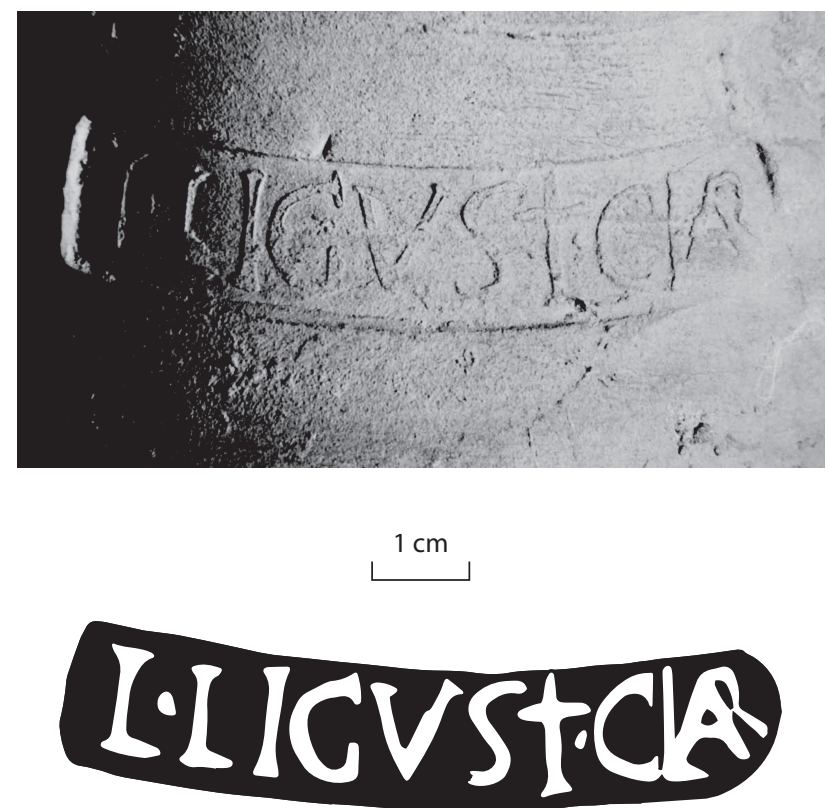

Fig. 9 : Anfora Dressel 26 eponima. Roma, Castro Pretorio. Mercati di Traiano, Inv. MT351. Particolare del bollo (foto Archivio SCBC; disegno M. Bonifay, A. Contino).

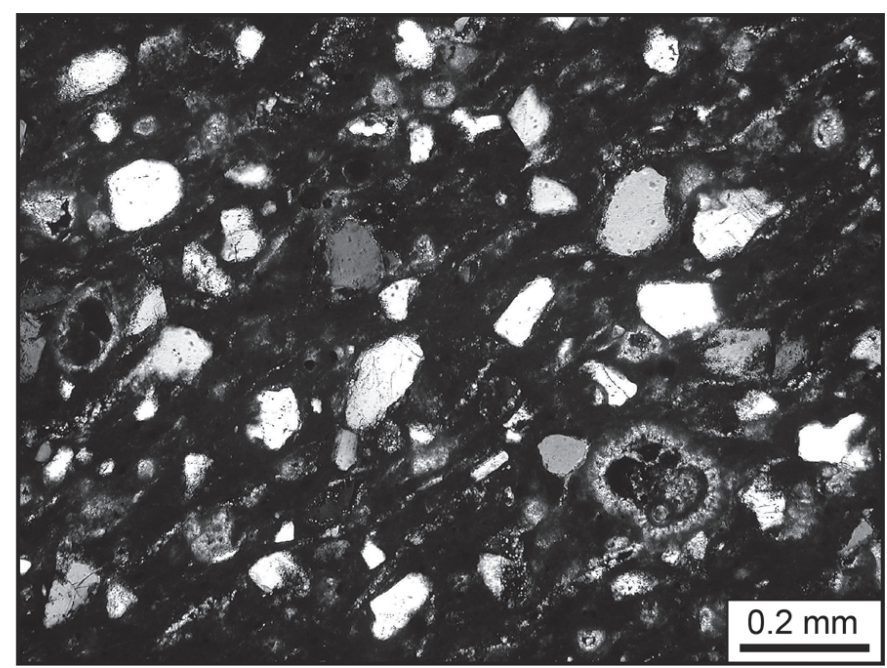

Fig. 10 : Anfora Dressel 26 eponima. Roma, Castro Pretorio. Mercati di Traiano, Inv. MT351 (analisi n. 11455). Microfotografie (Nx) (foto C. Capelli). 
datazione ancora più recente ${ }^{27}$. Il gentilizio è attestato infine in Africa a Lambesi in una lista di veterani di difficile datazione, ma collocabile tra la seconda metà del II e la prima metà del III secolo, sulla base delle attestazioni di gentilizi imperiali e toponimi africani ${ }^{28}$ (tab. 1).

Al momento non è possibile fornire ulteriori dati oltre la segnalazione della presenza di una gens Ligustia in Italia, Dalmazia e Africa. Non possiamo dunque identificare con certezza il personaggio citato nel bollo anforario né essere certi della sua origine. Purtroppo non è stato possibile rinvenire ulteriori attestazioni di L. Ligustius Clar(us) sulle due anfore Dressel 26.

\section{TITULI PICTI}

Sulla basa della lettura di alcuni tituli picti TAVR, VIR, MOL, STR presenti su contenitori del tipo Schoene XI conservati a Pompei, Theodor Peña proponeva per queste anfore un contenuto vinario e una provenienza siciliana tenendo conto che il titulus TAVR poteva indicare la città di Tauromenium (Taormina) ${ }^{29}$. Tuttavia, poiché come si è detto il tipo pompeiano raccoglie diversi contenitori, non è possibile stabilire se tutte le iscrizioni fossero attestate su anfore del tipo Dressel 26. Al momento sono stati individuati con certezza su tale contenitore solo i tituli TAVR e $\mathrm{MOL}^{30}$.

In un recente articolo collettivo sulle anfore Ostia LIX e XXXIII, a cui si rimanda per una disamina puntuale della lettura epigrafica ${ }^{31}$, A. Tchernia ha proposto una nuova interpretazione dei tituli, che sembrano indicare

27. La formula abbreviata " $D M$ " presente nell'epigrafe funeraria proveniente da Salona (CIL III, 3183a) è attestata dall'età flavia, ma soprattutto nel II secolo d.C., nelle province di Germania, Gallia e Illirico: Calabi Limentani 1991, p. 153-154.

28. L'epigrafe citata riporta i nomi dei veterani della legio III Augusta e talvolta il rango e la provenienza. Nel nostro caso è presente solo il nome Caius Ligustius. Si tratta quindi di un soldato di rango e provenienza sconosciuti. Secondo Le Bohec la lista si data al più tardi tra Settimio Severo e Massimino il Trace (193-238 d.C.), per la presenza del praenomen e del gentilizio Marci Aurelii, che rimandano agli imperatori Marco Aurelio, Commodo o Caracalla e per la presenza del praenomen e del gentilizio $Q$. Memmius, che evoca C. Memmius, legato del proconsole d'Africa nel 175-176. Per quanto riguarda la struttura dell'epigrafe, nel testo i soldati sono classificati per coorti e sono con buona probabilità tutti africani, anche in assenza del toponimo di provenienza. Fin dall'impero di Adriano infatti si cerca di reclutare su base locale nelle province. Secondo Cagnat, pur collocandosi nel corso del II secolo d.C., il testo rimane difficile da datare: CAgnat 1913, p. 297. Benché nel nostro caso la città di provenienza non sia conservata, le liste di legionari di stanza a Lambesi, successive al 166, riportano solo nomi di città africane o la formula "castris", per i nati nel campo. Ciò lascerebbe supporre che anche Caius Ligustius fosse di provenienza africana e che quindi la gens Ligustia prima attestata in Italia si sia poi in parte stabilita in Africa, fornendo soldati alla guarnigione della legio III Augusta di stanza a Lambesi.

29. PeÑA 2007.

30. Scotti 1984, tav. 165. CE 2017 (MOL) e Casa di Arianna CdA4339 (TAVR) inedito.

31. BONIFAY et alii 2015. la produzione e la lavorazione di olive di varie qualità, associata alla presenza dei tria nomina dei commercianti ${ }^{32}$. Per il titulus TAVR è stato proposta, in modo molto ipotetico, una lettura legata a un prodotto della città africana di Thabraca o Tauraca.

L'anfora del Castro Pretorio sembra tuttavia non presentare tituli né sul corpo né sul collo.

\section{ANALISI PETROGrafiche}

Ad un'analisi macroscopica l'anfora non sembrava presentare le caratteristiche né degli impasti tirrenici e della bassa Campania, né della Sicilia orientale, connessi alle presunte identificazioni dell'area di origine con le località di Venafro o di Taormina. Era invece possibile ipotizzare per il contenitore un'origine africana.

Una scheggiatura sul fondo dell'esemplare conservato ai Mercati di Traiano ha permesso il prelievo di un piccolo frammento, dal quale è stata realizzata una sezione sottile per lo studio petrografico.

Osservato al microscopio polarizzatore, l'impasto presenta una matrice argillosa principalmente ferrica, ossidata, e inclusioni abbondanti, piuttosto ben classate e fini $(<0.2 \mathrm{~mm})$, costituite essenzialmente da granuli di quarzo (angolosi o arrotondati, in parte probabilmente di origine eolica) e microfossili calcarei (foraminiferi) (fig. 10). La materia prima utilizzata (tal quale) è da riferire a sedimenti essenzialmente marini.

Tali caratteristiche permettono di accomunare, in linea generale, l'anfora in oggetto con tutti gli altri campioni di anfore Dressel 26 finora studiati ${ }^{33}$. Elemento comune di tutti gli impasti è la presenza dominante di quarzo, in parte eolico e spesso associato ad elementi carbonatici. Si deve notare tuttavia l'assenza di precisi confronti tessiturali con gli impasti di tali campioni, spesso più grossolani. Tali differenze sono forse correlabili con quelle morfologiche e/o cronologiche sopra discusse. Si potrebbe quindi ipotizzare la presenza di produzioni distinte nello spazio e/o nel tempo in una stessa regione.

L'assenza di componenti effusive alcalino-potassiche permette di scartare definitivamente l'ipotesi di una produzione localizzata nella fascia costiera campanolaziale (come pure nell'area di Venafro, caratterizzata da sequenze sedimentarie carbonatiche ${ }^{34}$ ). Le caratteristiche delle inclusioni delle Dressel 26 escludono inoltre una provenienza dalla Sicilia orientale, la cui geologia è dominata da rocce di natura sia vulcanica basica, sia metamorfica acida. L'abbondanza di elementi metamorfici è distintiva, in particolare, delle produzioni anforiche di $\operatorname{Naxos}^{35}$.

32. BONIFAY et alii 2015.

33. Contino, Capelli c.s.

34. Carta Geologica d'Italia, foglio 161 - Isernia (1971).

35. Franco, Capelli 2014, p. 548-549, 552. 


\begin{tabular}{|c|c|c|c|c|}
\hline & Personaggio & Tipologia iscrizione & Indicazioni specifiche & Bibliografia \\
\hline $\begin{array}{c}\text { Roma } \\
\text { (Castro Pretorio) }\end{array}$ & L. Ligusti Clari & Anfora Dressel 26 & & CIL XV, 3479 a, b \\
\hline Roma & C. Ligustius & Epigrafe funeraria & & NSA 1914, p. 397 \\
\hline Palestrina & C. Ligustius & Epigrafe onoraria (?) & $\begin{array}{c}\text { Magistri collegium } \\
\text { coronarium }\end{array}$ & CIL I, 3060 \\
\hline Capua & C. Ligustius & Epigrafe funeraria (?) & $\begin{array}{c}\text { Figli e liberti di } \\
\text { C. Ligustius }\end{array}$ & AE 1975, 209 (p. 216) \\
\hline Venafro & Ligustia Secunda & Epigrafe funeraria & CIL X 4934 \\
\hline Dalmazia & C. Ligustius & Epigrafe funeraria & & CIL III, 2417 \\
\hline Salona & C. Ligustius & Epigrafe funeraria & & CIL III, 3183a \\
\hline Salona & & & $\begin{array}{c}\text { Lista di veterani } \\
\text { Legio III Augusta }\end{array}$ & CIL VIII, 2569 \\
\hline Numidia & C. Ligustius & Epigrafe onoraria & \\
\hline Lambesi & &
\end{tabular}

Tab. 1 : Attestazione del gentilizio LIGVSTIVS (A. Contino).

Mentre gli impasti quarzosi (eolici) sono tipici della maggior parte delle produzioni africane ${ }^{36}$, e anche se non si può escludere del tutto un'origine dell'anfora Dressel 26 dalla Sicilia occidentale (non orientale) su basi geologiche, varie caratteristiche tipo-petrografiche, comuni ad altre varianti della forma e a tipi coevi, quali l'Africana Antica, suggeriscono piuttosto una provenienza africana.

La superficie presenta uno sbiancamento evidente caratteristico delle produzioni di anfore africane e dovuto all'utilizzo di acqua salmastra o salata ${ }^{37}$.

(C.C., A.C.)

\section{Contenuto}

In mancanza di analisi chimiche, sulla base della lettura epigrafica (v. supra), si propone per le anfore Dressel 26 un contenuto di olive o forse olio. Tuttavia ad oggi non si hanno certezze sul prodotto trasportato ${ }^{38}$.

(A.C.)

36. Capelli, Bonifay 2014.

37. Sull'uso di acqua salata per sbiancare le anfore africane cf. Peacock 1984.

38. BONIFAY et alii 2015.

\section{CONCLUSIONI:}

\section{PROVENIENZA DEL TIPO DRESSEL 26}

Lo studio tipo-petrografico condotto sull'anfora eponima del Castro Pretorio, comparato con quello di esemplari provenienti anche da altri contesti nell'ambito di una più ampia ricerca di cui non è possibile dar conto per esteso, ha permesso di raggiungere interessanti risultati in merito alla morfologia e provenienza del contenitore. I risultati tipopetrografici sembrano individuare alcune linee di continuità tra l'anfora Dressel 26 e l'anfora Africana Antica che potrebbe dunque essere la sua antecedente. La recente interpretazione di tituli attestati su anfore provenienti da Pompei ${ }^{39}$ pare escludere una attribuzione alla città di Taormina; pertanto è possibile proporre per la Dressel 26 una provenienza africana. In particolare l'esemplare del Castro Pretorio, pur differenziandosi in parte dalle principali famiglie petrografiche individuate per il tipo, presenta come queste elementi che permettono di individuarne l'origine sulla costa dell'Africa settentrionale. Alcune peculiarità d'impasto potrebbero essere giustificate con una specifica variante produttiva e/o cronologica, come sembrerebbe indicare anche l'analisi morfologica.

Per motivi archeologici, per l'assenza di elementi propri degli impasti noti di produzione tripolitana e byzacena e per l'affinità tra alcuni impasti di Dressel 26 e di Africane Antiche, si ipotizza che l'area principale di provenienza si possa collocare nella Tunisia nord-occidentale.

39. BONIFAY et alii 2015. 
Anche l'anfora Dressel 26 si inserisce dunque nella più ampia classe delle anfore Africane Precoci: i primi contenitori di forma romanizzata che appaiono in Africa, compresi tra la seconda metà del II a.C. e il II d.C. e articolati in diversi tipi (Africana Antica, Dressel 26, Ostia LIX e affini, Ostia XXIII).

La Tunisia nord-occidentale era nota in età romana ed in particolare tra l'età tardo-repubblicana e l'inizio dell'età imperiale, soprattutto per la produzione cerealicola, tuttavia le fonti testimoniano anche la presenza dell'arboricoltura ${ }^{40}$.
La classe della anfore africane precoci, di cui questo contenitore fa parte, costituirebbe un segno tangibile di questa produzione di derrate liquide rivolta anche all'esportazione. Per quanto attiene al contenuto al momento alcuni indizi sembrerebbero indicare per taluni contenitori la presenza di olive o olio, ma non possiamo del tutto escludere ad oggi un diverso prodotto o un uso polivalente delle anfore. Si tratterebbe ovviamente di scambi commerciali ancora occasionali e minoritari come suggerisce il basso numero di attestazioni.

(A.C., C.C., M.B.) 


\section{BIBLIOGRAFIA}

BONIFAY M. 2004, Études sur la céramique romaine tardive d'Afrique, Oxford (BAR Int. S. 1301).

Bonifay M. et alii 2015, Bonifay M., Botte E., Capelli C., Contino A., Duaoui D., Panella C., Tchernia A., "Nouvelles hypothèses sur l'origine et le contenu des amphores africaines Ostia LIX et XXIII", AntAfr 51, p. 189-210.

Calabi Limentani I. 1991, Epigrafia latina, Milano (Manuali Cisalpino 3).

CaGnat R. 1913, L'armée romaine d'Afrique et l'occupation militaire de l'Afrique sous les empereurs, Paris (2 édition).

Capelli C., Bonifay M. 2014, “Archéométrie et archéologie des céramiques africaines: une approche pluridisciplinaire, 2. Nouvelles données sur la céramique culinaire et les amphores", in N. Poulou-Papadimitriou, E. Nodarou, V. Kilikoglou (éd.), LRCW 4, Late Roman Coarse Ware, Cooking Wares and Amphorae in the Mediterranean: Archaeology and Archaeometry, The Mediterranean: A market without Frontiers, Oxford (BAR Int. S. 2616), p. 235-253.

Capelli C., Contino A. 2013, "Amphores tripolitaines anciennes ou amphores africaines anciennes ?", AntAfr 49, p. 199-208.

Carta M., Pohl I., Zevi F. 1978, Ostia. La Taberna dell'invidioso: Piazzale delle Corporazioni, portico ovest: saggi sotto i mosaici, (NSA, Suppl. alla s. VIII, vol. 32), Roma.

Contino A. 2013, "Tripolitana Antica e Dressel 26 a Roma. Il caso del Nuovo Mercato Testaccio. Dati preliminari", in L'Africa romana XIX, Roma, p. 1471-1487.

Contino A. 2015, Anfore africane tardorepubblicane e primoimperiali dal Nuovo Mercato di Testaccio a Roma (ipotesi di produzione, commercio e diffusione nel Mediterraneo), Tesi di dottorato presso Aix-Marseille Université e Università Cattolica di Milano, sotto la direzione di M. Bonifay e I. Romeo.

Contino A., CAPElli C. c.s., "Nuovi dati archeologici e archeometrici sulle anfore africane tardorepubblicane e primo imperiali: rinvenimenti da Roma (Nuovo Mercato Testaccio) e contesti di confronto", in Amphorae ex Hispania. Paisajes de producción y de consumo, Sociedad de Estudios de la Cerámica Antigua en Hispania III - ex officina hispana, Congreso de Tarragona (10-13 de diciembre de 2014).

Dressel H. 1879, "Di un grande deposito di anfore rinvenuto nel nuovo quartiere del Castro Pretorio", BCAR VII, s. II, p. 36-112, 143-196.

Empereur J.-Y., Hesnard A. 1987, "Les amphores hellénistiques du bassin occidental de la Méditerranée", in P. LÉvÊQUE, J.-P. Morel (éd.), Céramiques hellénistiques et romaines II, Paris (Annales littéraires de l'Université de Besançon 70 ; Centre de recherches d'histoire ancienne 331), p. 24-71.

Ferrandes A. 2008, "I contenitori da trasporto", in F. FilipPi (éd.), Horti et Sordes. Uno scavo alle falde del Gianicolo, Roma, p. $247-283$.

Ferrandes A. 2014, "Circolazione ceramica e approvvigionamento urbano a Roma nel I secolo a.C. Nuovi dati dall'area degli Horti Lamiani”, RCRF Acta 43, p. 353-366.
Franco C., CaPelli C. 2014, "New Archaeological and Archaeometric Data on Sicilian Wine Amphorae in the Roman Period $\left(1^{\text {st }}\right.$ to $6^{\text {th }}$ Century A.D.). Typology, Origin and Distribution in Selected Western Mediterranean Contexts", RCRF Acta 43, p. 547-556.

Geremia Nucci R. 2004-2005, "Il materiale ceramico", in F. Zevi, R. Geremia Nucci, A. Leone, "Ostia. Sondaggio stratigrafico in uno degli ambienti della domus dei Pesci (1995 e 1996)", NSA s. IX, XV-XVI, p. 50-246.

Hesnard A. 1980, "Un dépôt augustéen d'amphores à la Longarina (Ostie)", in J.H. D'Arms, E.C. KopfF (éd.), The Seaborne Commerce of Ancient Rome, Studies in Archaeology and History, Roma (Memoirs of the American Academy in Rome XXXVI), p. 141-156.

Lissi Caronna E. 1968, "Roma, Piccolo deposito di anfore in via Alessandro Nelli (tra il $14^{\circ}$ ed il $15^{\circ} \mathrm{km}$ della via Casilina)", NSA s. VIII, 22, p. 10-15.

LONG L. 1994, "Prospections et sondages archéologiques dans le Rhône, à Arles et ses environs", in Histoire du Rhône au Pays d'Arles, Actes du colloque (Arles, 7 novembre 1992), Arles, p. 45-71.

Ostia III, Carandini A., Panella C. (éd.) 1973, Le terme del Nuotatore. Scavo degli ambienti III, VI, VII. Scavo dell'ambiente $V$ e di un saggio nell'area SO, Roma (Studi Miscellanei 21).

Panella C. 1977, Anfore Tripolitane a Pompei, Quaderni di Cultura Materiale 1, p. 135-149.

PeAcock D.P.S. 1984, "Appendix 1, Seawater, Salt and Ceramics", in M.G. Fulford, D.P.S. Peacock (éd.), Excavations at Carthage: The British Mission, I. The Avenue du President Habib Bourguiba, Salammbo, The Pottery and Other Ceramic Objects from the Site, Sheffield, p. 263-264.

PeÑa J. T. 2007, "Two Groups of tituli picti from Pompeii and Environs: Sicilian Wine, not Flour and Hand-Picked Olives", JRA 20, p. 233-254.

Rizzo G. 2003, Instrumenta Urbis I, ceramiche fini da mensa, lucerne ed anfore a Roma nei primi due secoli dell'impero, Roma (CÉFR 307).

Rizzo G. 2014, "Le anfore, Ostia e i commerci mediterranei", in C. Panella, G. Rizzo (éd.), Ostia VI. Le terme del nuotatore. I saggi nell'area NE. Le Anfore, Ostia e i commerci mediterranei, Roma (Studi Miscellanei 38), p. 65-481.

Sciallano M., Sibella P. 1991, Amphores. Comment les identifier?, Aix-en-Provence.

Scotti C. 1984, "Anfore”, in M. Bonghi Jovino (éd.), Ricerche a Pompei: l'insula 5 della Regio VI dalle origini al 79 d.C, Roma, p. 281-292.

Ungaro L. et alii 2014, Ungaro L., Bernardini C., Giuffré M. T., Massera M., Milella M., Rivaroli L., Vigliarolo P., Vitti M., "Mercati di Traiano e Museo dei Fori Imperiali. La cisterna e le anfore (2013-2014)", BCAR CXV, p. 281-289.

Zevi F. 1966, "Appunti sulle anfore romane. I - La tavola tipologica del Dressel", ArchClass XVIII(2), p. 208-247.

Zevi F., Carta M. 1978 [1987], "La Taberna dell'invidioso", NSA 32, suppl., p. 9-164. 